\title{
Heat Transfer Enhancement Using Ferrofluids
}

\author{
Gary Rosengarten, Nicolette Gan, Cameron Stanley \\ RMIT University \\ School of Engineering, Melbourne, Australia \\ gary.rosengarten@rmit.edu.au; s3340640@student.rmit.edu.au; cameron.stanley@rmit.edu.au
}

\begin{abstract}
In this paper we demonstrate how ferrofluids (magnetic nanofluids) can be used to significantly enhance heat transfer rates in microchannels to help meet the ever-growing demand for high heat flux removal. There are a few mechanisms that can be used to aid mixing and to enhance heat transfer in inherently low Reynold number flow in microchannels. Firstly, the addition of a small amount of the thermally conductive paramagnetic particles can increase the thermal conductivity of the base fluid. For example, a solution of 5\% particles can increase the thermal conductivity by approximately $10 \%$, with only a $4 \%$ increase in dynamic viscosity. With the application of a magnetic field, the effective thermal conductivity can be increased further in the direction of the field, due to the formation of particle chains. Heat transfer can be additionally enhanced by applying a magnetic field to disrupt the flow, but a non-uniform magnetic field is required. Two-phase slug flow using a plug of ferrofluid in an immiscible liquid can have a large and controllable effect on the heat transfer rate.
\end{abstract}

Keywords: Ferrofluids, heat transfer, Two-phase flow, Microchannels.

\section{Introduction}

It is well documented that the amount of heat per unit area that needs to be removed in a range of devices is increasing significantly, almost in line with the exponential computing power increase described by Moore's law. As well as the increasing power density of integrated circuits, the increasing load requirements in power electronics, particularly for renewable energy is driving up the magnitude of heat that needs to be removed per unit area. In fact, some processes are being limited so as not to overheat. We are entering an era where standard cooling technologies involving air will not be able to remove enough heat. Thus, new mechanisms/systems with ultra-high heat flux removal capabilities need to be developed. However, there is a trade-off between increasing the heat removal rate and the energy required to achieve the heat removal.

One method to increase heat flux is to use microchannels [1]. These effectively increase the surface area available for internal convective flow. Additionally, even though the heat transfer coefficient can be very high in microchannels, it is still limited due to the flow being laminar because of the small Reynolds numbers associated with the small hydraulic diameter. Boiling in microchannels can offer very high heat transfer rates, but systems can suffer from flow instability and dry out in hot spots [2]. Other methods include using conducting nanofluids (but their usefulness and stability has been under question and as yet there are still no commercial applications), and non-phase changing two-phase slug flow that can offer increases in heat transfer by up to 500\% [3]. In this paper we look at using ferrofluids, nanofluids with paramagnetic nanoparticles coated with a surfactant layer dispersed in it, particularly as plugs in two-phase flow. Ferrofluids have a long commercial history, particularly in loud speakers [4] as a lubricant and damper. We focus on a novel method to use them as the disperse phase in two-phase microchannel heat sinks.

\section{Ferrofluids}

Commercial water-based ferrofluids (Domain Detection Kit, Ferrotec), containing EMG308, EMG408, EMG707, EMG708 and EMG807, were used to make diluted samples of 5\% and 10\% ferrofluid with de-ionized water (DIW). Thermophysical properties of the diluted samples were measured and compiled in Table 1 . The measurements indicate that due to the varying particle concentration and amount of surfactant, the properties change considerably. 
Table 1: Measured properties of diluted commercial ferrofluids.

\begin{tabular}{|c|c|c|c|c|c|}
\hline \multirow[t]{2}{*}{ Sample } & \multirow{2}{*}{$\begin{array}{c}\text { Magnetite } \\
\text { Content } \\
\text { (\% by Volume) }\end{array}$} & \multicolumn{4}{|c|}{ Measured Properties } \\
\hline & & $\begin{array}{l}\text { Density } \\
\left(\mathrm{kg} / \mathrm{m}^{3}\right)\end{array}$ & $\begin{array}{c}\text { Dynamic } \\
\text { Viscosity } \\
(\mathrm{mPa} \cdot \mathrm{s})\end{array}$ & $\begin{array}{l}\text { Contact } \\
\text { Angle }\left({ }^{\circ}\right)\end{array}$ & $\begin{array}{c}\text { Thermal } \\
\text { Conductivity } \\
(\mathrm{W} /(\mathrm{m} \cdot \mathrm{K}))\end{array}$ \\
\hline EMG308 5\% & $0.02-0.055$ & 1006.0 & 1.025 & 90 & 0.646 \\
\hline EMG308 10\% & $0.04-0.11$ & 1008.1 & 1.046 & 110 & 0.644 \\
\hline EMG408 5\% & $0.02-0.055$ & 1004.6 & 1.029 & 113 & 0.636 \\
\hline EMG408 10\% & $0.04-0.11$ & 1006.2 & 1.040 & 114 & 0.626 \\
\hline EMG707 5\% & $0.05-0.2$ & 1012.9 & 1.078 & 80 & 0.642 \\
\hline EMG707 10\% & $0.1-0.4$ & 1021.0 & 1.103 & 106 & 0.645 \\
\hline EMG707 20\% & $0.2-0.8$ & 1022.5 & 1.113 & 80 & 0.655 \\
\hline EMG708 5\% & $0.05-0.2$ & 1005.9 & 1.029 & 109 & 0.682 \\
\hline EMG708 10\% & $0.1-0.4$ & 1009.8 & 1.060 & 105 & 0.684 \\
\hline EMG807 5\% & $0.05-0.4$ & 1006.7 & 1.040 & 100 & 0.644 \\
\hline EMG807 10\% & $0.1-0.8$ & 1009.7 & 1.050 & 84 & 0.651 \\
\hline DIW & - & 1000.0 & 1.000 & 115 & 0.610 \\
\hline
\end{tabular}

\subsection{Properties with a magnetic field}

The properties of the different types of ferrofluids were also measured in the presence of a magnetic field and the results are presented in Fig. 1. Clearly, both the viscosity and thermal conductivity increase with increased magnetic field strength. The viscosity was measured in a capillary viscometer with the field perpendicular to the flow direction. The magnetic field caused the particles to align perpendicularly to the flow direction, creating an increased resistance to flow. Similarly, the thermal conductivity was measured using a the KD2 Pro Thermal Property Analyzer (Decagon Devices Inc.), which is based on the transient hot-wire method. The field was applied perpendicular to the axis of the probe, so the formed particle chains helped increase the effective conductivity.
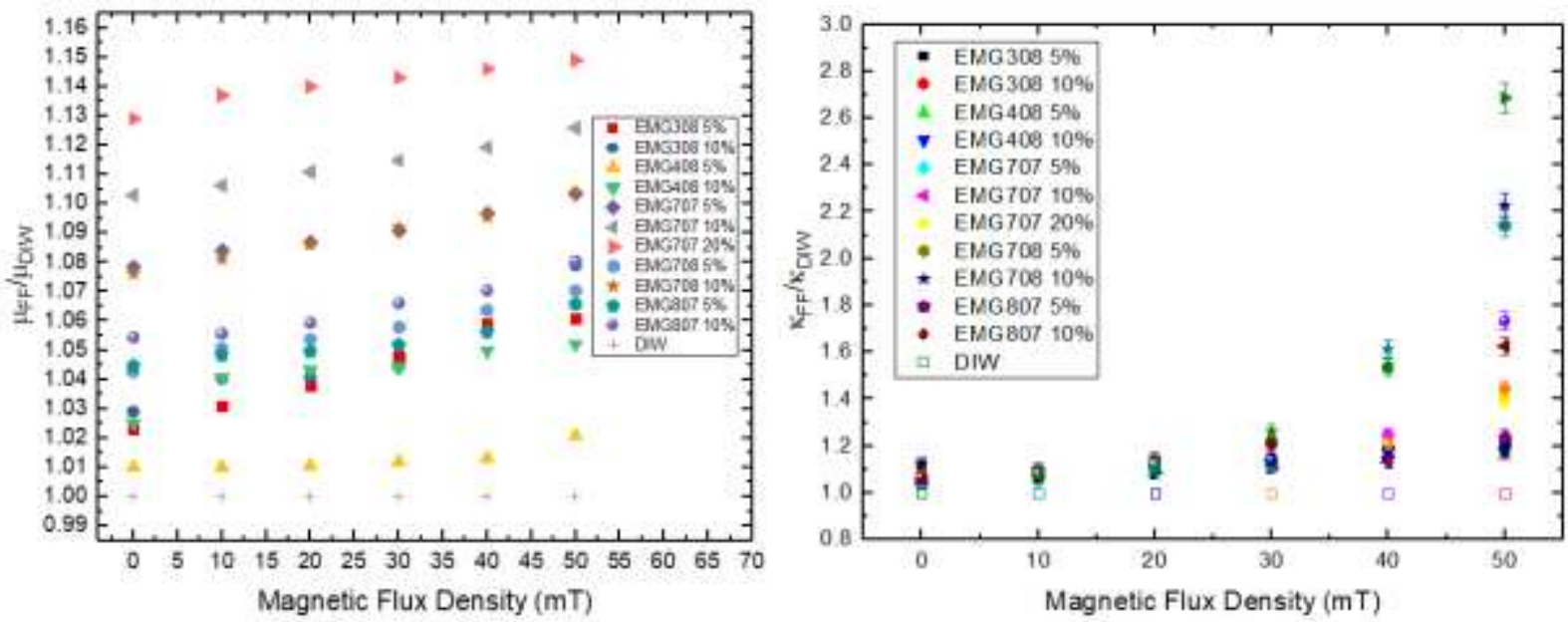

Fig. 1: Relative viscosity (L) and thermal conductivity (R) (to deionized water) of the various ferrofluids as a function of applied magnetic field at $25^{\circ} \mathrm{C}$ (adapted from [5]). 


\section{Experimental Set-up}

An inverse sacrificial mould of the channel was 3D printed in acrylonitrile butadiene styrene (ABS). A nichrome wire was wrapped around the mould, and various thermocouples (TC) were attached to it. It was then immersed in polydimethylsiloxane (PDMS) and cured, after which the ABS was dissolved away [5] leaving the sealed channel with heater and TCs embedded, as shown in Fig. 2Fig. 2. This gave us enough information to calculate the Nusselt number defined as $N u=h D / k$, where $\mathrm{D}$ is the hydraulic diameter of the channel, $\mathrm{k}$ is the thermal conductivity of the ferrofluid and $\mathrm{h}$ is the convective heat transfer coefficient given by $h=\dot{m} c_{p} /\left(T_{\text {wall,mean }}-T_{\text {fluid,mean }}\right)$.

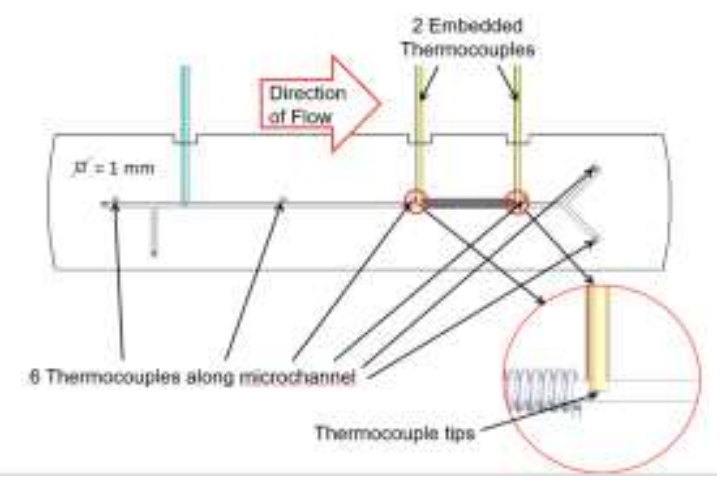

Fig. 2: Schematic of channel layout.

\section{Single Phase Ferrofluid heat transfer}

The relative (to deionized water) Nusselt number for single-phase ferrofluid flow with a uniform magnetic field is plotted in Fig. 3. The error bars represent the combined uncertainty of the measurements (including temperature, flow rate and channel size) to a 95\% confidence level. The results show a decreasing heat transfer rate as the magnetic field is increased. To investigate the mechanism behind this trend, we visualised the flow which showed that the magnetic field caused the particles to separate and accumulate on the wall. Assuming the bulk fluid concentration becomes zero in the particle depletion region (centre of the channel), and that the accumulated region thermal conductivity increases to the value measured in steady state experiments (Fig. 1. 1), a simple thickness weighted thermal conductivity calculation shows how the conductivity decreases.
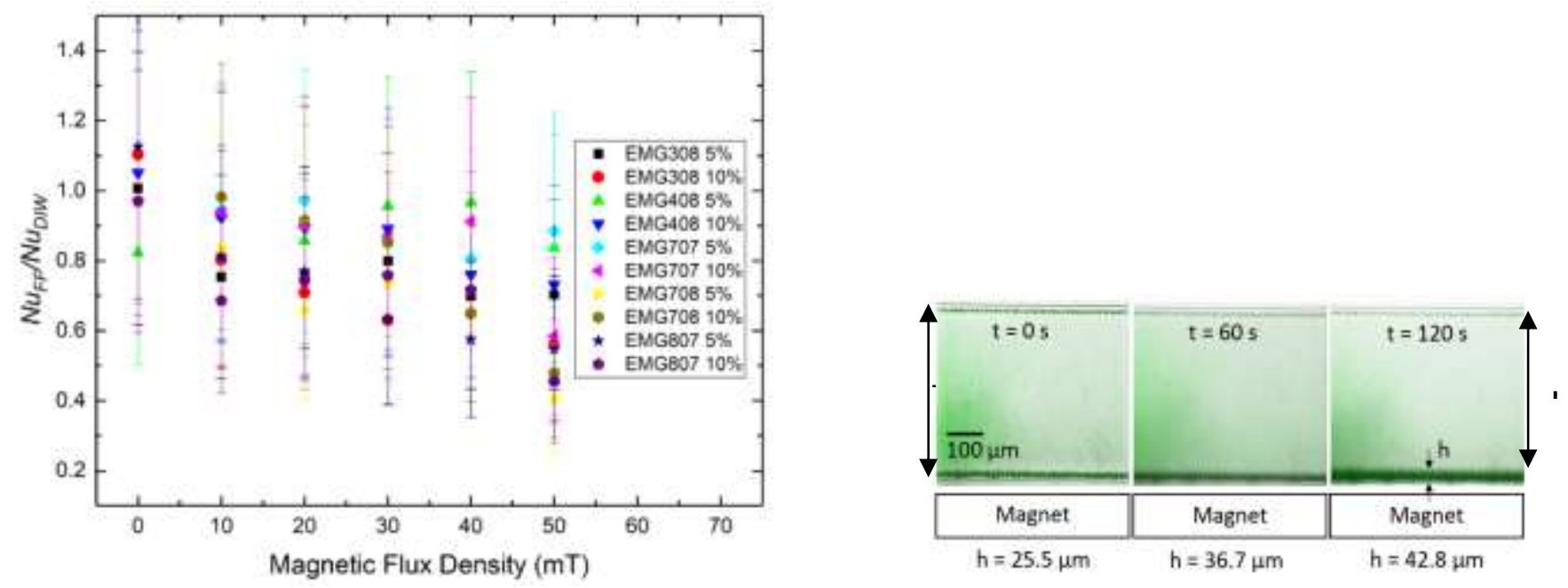

Fig. 3: Left; $N u_{\mathrm{FF}} / N u_{\mathrm{DIW}}$ vs Magnetic Flux Density @ $50 \mathrm{~mL} / \mathrm{h}$ and, Right; Visualisation of particles separating from the bulk fluid under the influence of the magnetic field (adapted from [5]). 


\section{Multiphase slug flow}

Slug flow, will not only increase the heat transfer rate due to the extra mixing associated with the shear forces around the slug, but if the slugs are made of ferrofluids the particles cannot agglomerate at the wall. Slugs were formed by pumping the ferrofluid via a T-junction into flowing $0.65 \mathrm{cSt}$ silicone oil as shown in Fig. 4a. The image of a slug is shown in Fig. 4b without the magnetic field and in Fig. $4 \mathrm{c}$ with the magnetic field, show how the particles align with the magnetic field in the slug, yet due to the strong surface tension forces the slug shape is not significantly deformed.
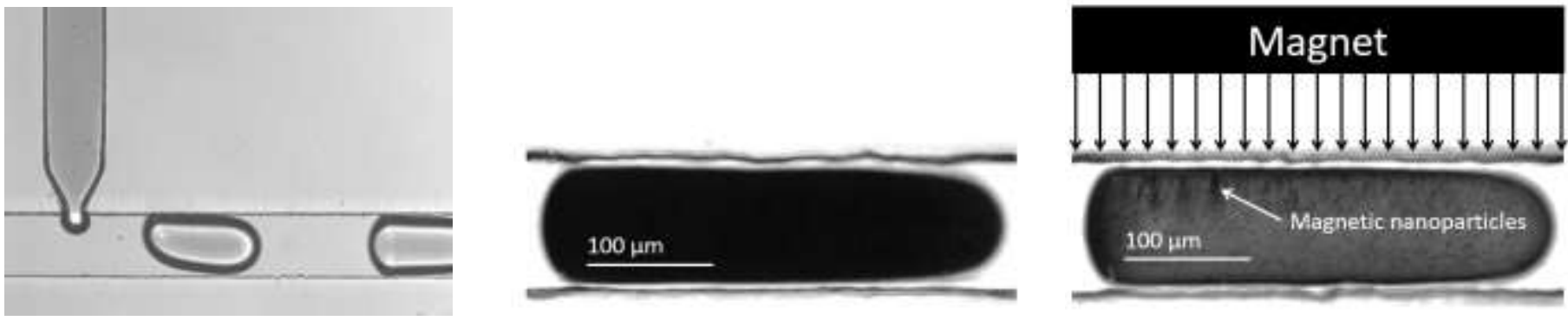

Fig. 4: a) Ferrofluid slug formation using a T-junction b) Individual slug without a magnetic field c) Individual ferrofluid slug

Fig 5. shows the variation of Nusselt number with magnetic field for both single phase and two-phase flow. Unlike single phase flow, the two phase flow increase with applied magnetic field. This appears to be primarily the effect on the slug formation process which makes the slugs slightly longer thus increasing the heat transfer rate. There does not appear to be a inherent extra mixing effect in the thermal boundary layer to increase the heat transfer rate.

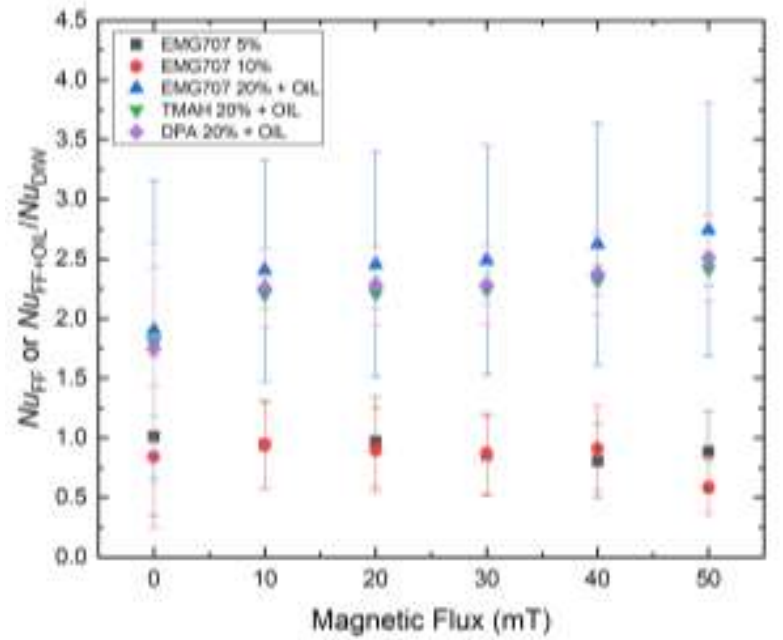

Fig. 5: Relative Nusselt number as a function of applied magnetic field @ $50 \mathrm{~mL} / \mathrm{h}$ for both single and two-phase flow

\section{Conclusion}

We have shown that in microchannels, even with uniform magnetic flux, the flow and heat transfer rate can be significantly affected by the interaction of the ferrofluid and the magnetic field both in single-phase and two-phase flow and we give some insight into the process. Further work will be carried on using multiple magnets to form and nonuniform magnetic field to disrupt the thermal boundary layer. 


\section{Acknowledgements}

The authors acknowledge funding through the Australian Research Council Linkage grant LP150100153.

\section{References}

[1] T. Dixit and I. Ghosh, "Review of micro-and mini-channel heat sinks and heat exchangers for single phase fluids," Renewable and Sustainable Energy Reviews, vol. 41, pp. 1298-1311, 2015.

[2] Y. K. Prajapati and P. Bhandari, "Flow boiling instabilities in microchannels and their promising solutions-A review," Experimental Thermal and Fluid Science, vol. 88, pp. 576-593, 2017.

[3] T. Bandara, N. T. Nguyen, and G. Rosengarten, "Slug flow heat transfer without phase change in microchannels: A review," Chemical Engineering Science, vol. 126, pp. 283-295, 2015.

[4] R. Rosensweig, Y. Hirota, S. Tsuda, and K. Raj, "Study of audio speakers containing ferrofluid," Journal of Physics: Condensed Matter, vol. 20, no. 20, pp. 204147, 2008.

[5] N. G. J. Gui, C. Stanley, N.-T. Nguyen, and G. Rosengarten, "Ferrofluids for heat transfer enhancement under an external magnetic field," International Journal of Heat and Mass Transfer, vol. 123, pp. 110-121, 2018. 